\title{
RISCO DE LESÃO POR PRESSÃO E NÍVEIS SÉRICOS DE VITAMINA C E GLICEMIAS EM HOSPITALIZADOS
}

\section{Patricia Leticia Beche ${ }^{1}$; Mairin Schott ${ }^{2}$; Sérgio Mortari ${ }^{3}$; Clandio Marques ${ }^{4}$; Elisângela Colpo 5}

\section{RESUMO}

Pessoas com Acidente Vascular Cerebral (AVC) podem ser acometidos por Lesão por Pressão (LPP), aumentando o risco de complicações durante a hospitalização. Objetivou-se associar o risco de desenvolver LPP com os níveis séricos de vitamina C e glicemia, em hospitalizados com AVC. Foi coletado dados como idade, gênero, doenças associadas e glicemias, além da Escala de Braden e níveis séricos de vitamina $\mathrm{C}$, que foram determinados por Cromatografia à Líquido de Alta Eficiência (CLAE). As diferenças foram consideradas significativas quando $p<0,05$. Não foram observadas diferenças significativas em relação as médias das glicemias e vitamina $C$ sérica com o risco de LPP $(p>0,05)$. No entanto, observou-se valores de hiperglicemia maiores em pacientes com maior risco de LPP. Além disso, os pacientes independentes do risco de desenvolver LPP apresentaram níveis séricos de vitamina C adequados. Conclui-se que os parâmetros avaliados não influenciaram no risco de desenvolvimento da LPP.

Palavras-chave: Cicatrização; Escala de Braden; Hiperglicemias.

Eixo Temático: Atenção Integral e Promoção à Saúde.

\section{INTRODUÇÃO}

O Acidente Vascular Cerebral (AVC) é definido pela interrupção de fluxo sanguíneo para o cérebro, com consequente obstrução ou rompimento de vasos sanguíneos cerebrais. Existem dois tipos de AVC, o AVC isquêmico (AVCi) que

\footnotetext{
${ }^{1}$ Nutricionista pela Universidade Franciscana - UFN. Email: patricia.beche77@gmail.com

2 Nutricionista pela Universidade Franciscana - UFN. Doutoranda em Ciências Biológicas: Bioquímica Toxicológica pela UFSM. E-mail: mairinschott@hotmail.com

${ }^{3}$ Professor do Curso de Biomedicina e do Mestrado em Nanociências da Universidade Franciscana UFN. Email: mortari@ufn.edu.br

${ }^{4}$ Professor da Universidade Franciscana - UFN. Email: clandiomarques@gmail.com

${ }^{5}$ Professora do Curso de Nutrição e do Mestrado em Ciências e Saúde da Vida da Universidade Franciscana - UFN. elicolpo@ufn.edu.br
} 
ocorre quando há obstrução de uma artéria, que impossibilita a passagem de oxigênio para as células do cérebro, podendo ocorrer por causa de um trombo ou um êmbolo, sendo o mais prevalente todos os casos. Já o AVC hemorrágico (AVCh) acontece quando há um rompimento de vaso cerebral, resultando em extravasamento de sangue no cérebro, ocasionando aneurisma ou trauma (MINISTÉRIO DA SAÚDE, 2021; REDE BRASIL, 2021; WHO, 2018).

A maioria dos indivíduos com doenças neurológicas encontram-se acamados, impossibilitados de aliviar a pressão sobre os ossos, sendo frequentemente acometidos por Lesão Por Pressão (LPP). Ela é definida como alteração localizada na pele, membranas, mucosas ou tecidos subjacentes, causada pela pressão prolongada sobre a pele. Alguns dos fatores que contribuem para o seu aparecimento são Hipertenção Arterial Sistêmica (HAS), Diabetes Mellitus (DM) e inconsciência (COX; RASMUSSEN, 2014; HAJHOSSEINI; LONGAKER; GURTNER, 2020; LEE et al., 2016).

O DM é o segundo principal fator de risco do AVC, por piorar a HAS e contribuir na formação da aterosclerose, além de contribuir para o desenvolvimento da LPP (CERRIELLO et al., 2013). Além disso, a hiperglicemia pode diminuir a captação de vitamina $C$ pelas células intestinais, interferindo na formação de colágeno e, consequentemente prejudicar a cicatrização da LPP (COPER et al., 2013).

O estudo justifica-se em avaliar se os níveis séricos de vitamina $C$ e hiperglicemias poderiam aumentar o risco de desenvolver LPP. Desta forma, o estudo teve como objetivo associar o risco de desenvolver lesão por pressão com os níveis séricos de vitamina $\mathrm{C}$ e glicemia em pacientes hospitalizados com AVC.

\section{METODOLOGIA}

O trabalho a seguir trata-se de um estudo transversal. Os dados da pesquisa foram coletados em um hospital público da região central do Rio Grande do Sul, Brasil, durante o período de agosto de 2018 a agosto de 2019. A amostra foi por conveniência, composta por adultos e idosos hospitalizados pós-AVC, de ambos os gêneros. O estudo foi aprovado pelo Comitê de Ética e Pesquisa e está registrado 
sob o número 1.579.090, conforme a Resolução n. 466/12 do Conselho Nacional de Saúde, referente a pesquisas com seres humanos. O termo de consentimento livre e esclarecido (TCLE) foi obtido por escrito de todos os participantes.

As coletas de dados como idade, gênero, motivo da internação, doenças associadas e glicemias foram coletadas a partir do prontuário eletrônico dos pacientes. Já os dados para avaliar o risco de desenvolvimento da LPP foram coletados a partir do registro da Escala de Braden pela equipe da enfermagem do hospital. A versão brasileira validada da Escala de Braden foi utilizada neste estudo (PARANHOS; SANTO, 1999), e classsificada de acordo com o grau de gravidade, sendo categorizadas da seguinte maneira: escore $\geq 15$ baixo risco; escore entre 13 a 14 risco moderado; escore $\leq 12$ risco alto (AYELLO; BRADEN, 2002).

Para classificacação das glicemais, foi considerado hiperglicemia quando valores maiores que $140 \mathrm{mg} / \mathrm{dL}$ (SOCIEDADE BRASILEIRA DE DIABETES, 2019). Para realização da análise da vitamina $C$ sérica as amostras de sangue foram adquiridas a partir da sobra dos tubos de coletas destinados para as análises sanguíneas dos pacientes durante a hospitalização sendo, posteriormente, armazenadas a $-80^{\circ} \mathrm{C}$. As amostras foram precipitadas com TCA 5\% (1:1), homogeneizadas com o auxílio do vórtex e centrifugadas à $3000 \mathrm{RPM}$ a $10^{\circ} \mathrm{C}$ por 10 minutos. $A$ análise de vitamina $C$ sérica foi realizada Cromatografia à Líquido de Alta Eficiência (CLAE) sendo utilizada uma coluna de cromatográfica C18 $(15 \mathrm{~mm} \times$

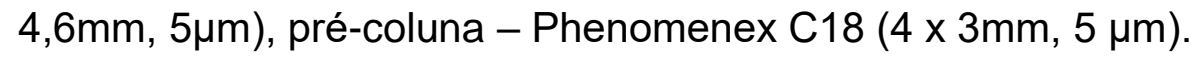

Em relação aos valores de vitamina $C$ sérica foram considerados valores maiores ou iguais a $28 \mu \mathrm{mol} / \mathrm{L}$ como normais, e valores abaixo de $28 \mu \mathrm{mol} / \mathrm{L}$ são definidos em depleção de vitamina $C$. Já os valores séricos abaixo de $11 \mu \mathrm{mol} / \mathrm{L}$ são definidos em deficiência de vitamina C (JOHNSTON; CORTE, 1999).

Posteriormente, os dados obtidos foram tabulados e armazenados em uma tabela no programa Microsoft Excel. Para a caracterização da amostra foi realizado uma análise descritiva dos dados dos participantes, sendo que as variáveis categóricas foram apresentadas em forma percentual e as quantitativas em forma de média e desvio padrão. 
Verificou-se a normalidade das variáveis através do teste de Shapiro-Wilk. Na análise das variáveis quantitativas foi utilizado Anova ou Kruskal-Wallis na comparação de 3 grupos, dependendo do resultado da normalidade. As diferenças foram consideradas significativas quando os resultados apresentaram o valor- $p<$ 0,05. O software IBM SPSS Versão 25 foi utilizado como ferramenta computacional para a análise estatística dos dados.

\section{RESULTADOS E DISCUSSÕES}

O estudo contou com a participação de 64 pacientes hospitalizados com AVC. Com média de idade de $64 \pm 13,12$ anos, sendo a maioria do gênero masculino e idosos, em relação ao tipo de AVC, a maior parte da amostra apresentou AVC do tipo isquêmico, sem Diabetes Mellitus. Dos pacientes $48,4 \%$ apresentaram alto risco de desenvolver LPP (Tabela 1).

Tabela 1: Características sóciodemográficas em pacientes hospitalizados com AVC:

\section{Dados Sociodemográficos}

\section{Pacientes $\mathrm{n}=64$}

$\mathrm{n}(\%)$

\begin{tabular}{lc}
\hline Gênero & $37(57,8)$ \\
Masculino & $27(42,2)$ \\
Feminino & $21(32,8)$ \\
\hline Idade & $43(67,2)$ \\
Adultos & \\
Idosos & $46(72)$ \\
\hline Tipo de AVC & $18(28)$ \\
AVCi & \\
AVCh & $26(40,6)$ \\
\hline Diabetes Mellitus (DM) & $38(59,4)$ \\
Com DM & \\
Sem DM & $21(32,85)$ \\
\hline Risco de desenvolver LPP & $12(18,75)$ \\
Risco baixo & $31(48,4)$ \\
Risco moderado & \\
Risco alto & \\
\hline
\end{tabular}

$\mathrm{Na}$ tabela 2 não foram observadas diferenças significativas em relação aos valores médios das glicemias e de vitamina $C$ sérica com o risco de LPP ( $p>0,05)$. 
Se observa que em relação a vitamina $\mathrm{C}$ todos os pacientes independentes do risco de desenvolver LPP apresentaram níveis séricos adequados, ou seja, maiores que $28 \mu \mathrm{mol} / \mathrm{L}$. Entretanto, em relação aos valores médios de glicemias, os pacientes com risco baixo e alto de desenvolver LPP apresentam hiperglicemias, segundo a Sociedade Brasileira de Diabetes (2019).

Tabela 2: variáveis em pacientes com AVC e seu risco de desenvolver LPP, conforme as classificações da Escala de Braden.

\section{LPP}

Marcadores

Risco Baixo Risco Moderado Risco Alto
p

Vit. C sérica ( $\mu \mathrm{mol} / \mathbf{L}) \quad 247,35 \pm 187,12 \quad 370,30 \pm 223,13 \quad 328,46 \pm 177,95 \quad 0,157$

Glicemias (mg/dL) $\quad 142,40 \pm 42,30 \quad 137,28 \pm 41,84 \quad 151,11 \pm 49,14 \quad 0,889$
LPP: Lesão por pressão

A vitamina $C$ desempenha vários papéis no corpo humano, entre eles o estímulo da síntese de colágeno, para consequente cicatrização de feridas (CHAMBIAL et al., 2013), entretanto, as hiperglicemias podem diminuir a captação de vitamina $\mathrm{C}$, prejudicando a formação de colágeno $\mathrm{e}$, consequentemente prejudicar a cicatrização da LPP. A hiperglicemia também pode prejudicar o processo de fagocitose e migração dos leucócitos para o local da ferida, dificultando a cicatrização pelas oscilações glicêmicas (CERRIELLO et al., 2013; COPER et al., 2013).

Em nível celular, o aumento nos níveis da glicose sanguínea resulta em dano mitocondrial pela geração de espécies reativas de oxigênio (EROS) em disfunção endotelial pela inibição da produção de óxido nítrico. A hiperglicemia aumenta os níveis de citocinas, que estão associadas a maiores taxas de mortalidade e complicações infecciosas (COPER et al., 2013).

\section{CONCLUSÃO}

Conclui-se que níveis séricos de vitamina C e glicemias de pacientes hospitalizados com AVC não influenciaram no risco aumentado de desenvolvimento da lesão por pressão. 


\section{AGRADECIMENTOS}

A UFN pela bolsa recebida.

\section{REFERÊNCIAS}

AYELLO, Elizabeth A.; BRADEN, Barbara. How and Why to Do Pressure Ulcer Risk Assessment. Advances, [s. I.], v. 15, n. 3, p. 125-133, 2002.

CERRIELLO, et al. Vitamin C further improves the protective effect of GLP-1 on the ischemia-reperfusion-like effect induced by hyperglycemia post-hypoglycemia in type 1 diabetes. Cardiovascular Diabetology, v.12, p.97, 2013.

CHAMBIAL, Shailja et al. Vitamin $\mathrm{C}$ in disease prevention and cure: An overview. Indian Journal of Clinical Biochemistry, [s. I.], v. 28, n. 4, p. 314-328, 2013.

COPER, C. P., et al. Intestinal Dehydroascorbic Acid (DHA) Transport Mediated by the Facilitative Sugar Transporters, GLUT2 and GLUT8. The Journal of Biological Chemistry, v. 288, n. 13, p. 9092-9101, 2013.

COX, Jill; RASMUSSEN, Louisa. Enteral nutrition in the prevention and treatment of pressure ulcers in adult critical care patients. Critical Care Nurse, [s. I.], v. 34, n. 6 , p. 15-27, 2014.

HAJHOSSEINI, Babak; LONGAKER, Michael T.; GURTNER, Geoffrey C. Pressure Injury. Annals of Surgery, [s. I.], v. 271, n. 4, p. 671-679, 2020.

JOHNSTON, CAROL S; CORTE, CORINNE. People with Marginal Vitamin C Status are at High Risk of Developing Vitamin C Deficiency. Journal of the American Dietetic Association, [s. I.], v. 99, n. 7, p. 854-856, 1999.

LEE, Shang Yi et al. Outcomes after Stroke in Patients with Previous Pressure Ulcer: A Nationwide Matched Retrospective Cohort Study. Journal of Stroke and Cerebrovascular Diseases, [s. I.], v. 25, n. 1, p. 220-227, 2016.

MINISTÉRIO DA SAÚDE. O que é o AVC?. Disponível em: https://www.gov.br/saude/pt-br/assuntos/saude-de-a-a-z-1/a/avc-0-que-e-causassintomas-tratamentos-diagnostico-e-prevencao. Acesso em: 18 mai. 2021.

PARANHOS, Wana Yeda; SANTO, Vera Lúcia C.G. Avaliação de risco para úlceras de pressão por meio da Escala de Braden, na língua portuguesa. Rev esc enferm USP, [s. I.], v. 33, n. 1, p. 191-206, 1999.

REDE BRASIL AVC. O que é o AVC?. Disponível em: http://www.redebrasilavc.org.br/para-pacientes-e-falimiares/o-que-e-avc/. Acesso em: 23 mar. 2021. 
SOCIEDADE BRASILEIRA DE DIABETES. Diretrizes da Sociedade Brasileira de Diabetes: 2019-2020. São Paulo: Editora Clannad, 491 p, 2019.

WORLD HEALTH ORGANIZATION. Stroke, cerebrovascular accident. Disponível em: http://www.worldstrokecampaign.org/pt br/learn/types-of-stroke.html.Acesso em: 05 jun. 2018. 